\title{
New, Efficient and Robust, Fiber-Based Quantum Key Distribution Schemes
}

\author{
W.T. Buttler, J. R. Torgerson and S. K. Lamoreaux \\ University of California, Los Alamos National Laboratory, Los Alamos, New Mexico 87545
}

(November 1, 2018)

\begin{abstract}
We present a new fiber based quantum key distribution (QKD) scheme which can be regarded as a modification of an idea proposed by Inoue, Waks and Yamamoto (IWY) [1]. The scheme described here uses a single phase modulator and two differential delay elements in series at the transmitter that form an interferometer when combined with a third differential delay element at the receiver. The protocol is characterized by a high efficiency, reduced exposure to an attack by an eavesdropper, and higher sensitivity to such an attack when compared to other QKD schemes. For example, the efficiency with which transmitted data contribute to the private key is $3 / 4$ compared with $1 / 4$ for BB84 22]. Moreover, an eavesdropper can aquire a maximum of $1 / 3$ of the key which leads to an error probability in the private key of $1 / 3$. This can be compared to $1 / 2$ and $1 / 4$ for these same parameters in both BB84 and IWY. The combination of these considerations should lead to increased range and key distribution rate over present fiber-based QKD schemes.
\end{abstract}

PACS Numbers: 03.67.-a, 03.67.Hk, 03.67.Dd, 42.79.Sz

The quantum key distribution (QKD) scheme recently proposed by Inoue, Waks and Yamamoto [1] represents a new concept that should prove to be a significant advance for fiber-based QKD. Their scheme, which we label IWY, is sketched in Fig. 11 for the case of a two delayelement transmitter. Essentially, a single pulse entering the transmitter leaves as a superposition of three pulses, the last two of which have been given a random phase shift of $\Delta \phi \in\{0, \pi\}$ relative to the first in addition to a fixed time delay of $\Delta t$ and $2 \Delta t$ relative the time associated with the input state: The photon pulse is superposed in time and phase. The receiver effects a time delay of $\Delta t$ and causes the superposed pulses to overlap and hence interfere in the same manner as superposed pulses in the standard Franson interferometer [3] used in BB84-like fiber QKD as shown in Fig. 3. (The transmitter and receiver are typically referred to as Alice $(\mathbf{A})$ and Bob (B) within the quantum information community.)

The advantages of this new scheme are numerous: (I) the efficiency with which transmitted data contribute to the private key, which we label $\eta_{p}$ the "protocol efficiency," is increased to $\eta_{p}=1-1 / N$, where $N$ is the number of temporal superpositions leaving the transmitter; (II) the amount of information an eavesdropper (E, usually Eve) can acquire on the key is reduced as $N$ increases; (III) the maximum error probability caused by eavesdropping, which we name "disturbance" and label as $p_{d}$, increases with $N$ and allows error reconciliation to be performed on data with a higher initial error probability; and (IV) $\mathbf{B}$ does not need a phase-modulator (PM).

The implications of these advantages, relating to the points raised above, are that $\mathbf{A}$ and $\mathbf{B}$ generate more key and can tolerate and correct for higher error rates because: (i) qubits accumulate at a higher rate; (ii) the fraction of key leaked during eavesdropping, $\eta_{e}$, that must be discarded to ensure the privacy is reduced with increasing $N\left(\eta_{e}\right.$ relates the maximum amount of information $\mathbf{E}$ can acquire on the quantum key in a specified attack on the quantum channel); (iii) the liklihood of detecting E's attacks on the quantum channel is increased as her eavesdropping creates a larger disturbance within the qubits; and (iv) the random choice of $\Delta \phi$ by $\mathbf{B}$ necessary in other schemes is eliminated in IWY and yields an increase in $\eta_{p}$ over these other schemes $\left(\eta_{p}\right.$ increases more than $300 \%$ for the case of the typical, or current, implementations of fiber based BB84, e.g. as shown in Fig. 3).

A further consequence of removing the PM from the receiver is that the typical 3-6 dB loss in PMs is removed from B's receiver. Thus, the key rate, robustness against errors, and distance over which qubits can be transmitted can be enhanced over currently realized fiber based QKD systems.

The scheme we introduce here, and labelled BLT and shown in Fig. 2 for two transmitter delay elements, improves upon IWY in several significant ways while retaining all of IWY's advantages over other schemes. First, BLT is simpler to implement because the series-element construction of BLT can be achieved with simple 50/50 beamsplitters (BSs), while the parallel construction of IWY requires more specialized BSs to balance the output pulses' intensities. Second, the efficiency $\left(\eta_{p}\right)$, potential eavesdropper knowledge $\left(\eta_{e}\right)$ and disturbance $\left(p_{d}\right)$ all favor BLT over IWY for the same number of delay elements in the transmitter. In BLT, the number $N$ of superposed pulses increases as $N=2^{m}$ where $m$ is the number of delay elements at the transmitter; in IWY $N$ increases as $N=m+1$. For example, the serial scheme of BLT yields $\eta_{p+} \geq 3 / 4$ while for the parallel scheme of IWY $\eta_{p \|} \geq 2 / 3$ with the lower bound given by $m=2$ in each case. Moreover, the eavedropper's potential information $\eta_{e}$ is less in BLT than in IWY, and the disturbance $p_{d}$ is greater in BLT than in IWY, regardless of the type of attack employed by E. Third, a single PM outside the transmitter delay elements further reduces the com- 
plexity and expense of the system and can increase its efficiency. For example, it is well-known that QKD is more secure and more efficient if each superposition of pulses contains exactly one photon. This would most likely be realized with a single photon source [4] before the delay elements and appropriately modified optics as discussed below. A single PM outside the transmitter delay elements dramatically increases the receiver efficiency in this situation, again due to the typical 3-6 dB loss of PMs.

Consider a fiber BB84 system as shown in Fig. 3 in which A sends single, or weak coherent, photon states to $\mathbf{B}$ 3.5.6. For each input pulse the phase of $\mathbf{A}$ 's $\mathrm{PM}$ is randomly toggled among a choice of four phases: $\phi_{A} \in\{0, \pi / 2, \pi, 3 \pi / 2\}$. As the pulses arrive at $\mathbf{B}$, the PM at that end is toggled randomly between $\phi_{B} \in\{0, \pi / 2\}$ for each superposition of pulses to select the measurement basis for that superposition. (B's delay element is adjusted so that a phase difference $\phi_{A}-\phi_{B}=0$ causes always a photodetection at the same detector and $\phi_{A}-\phi_{B}=\pi$ causes always a photodetection at the other detector.) After the weak pulses are exchanged $\mathbf{B}$ reveals the times when photodetections were observed and the measurement bases chosen at those times. A notes when their phases satisfied $\phi_{A}-\phi_{B} \in\{0, \pi\}$, and tells $\mathbf{B}$ to discard measurement results which were not collected when this condition was satified. What remains is potentially secret key in which $\phi_{A}-\phi_{B} \in\{0, \pi\}$ correspond to binary values $\{0,1\}$.

The exchanged qubits will undoubtedly contain errors which must be eliminated through an appropriate error reconciliation protocol 7.8]. After error reconciliation, privacy amplification [9] - which is linked to the error rate - must be applied to the remaining error-free key to reduce E's information to an acceptable level. If the error rate is too high all of the remaining key bits may be eliminated during this phase. In addition, the channel must be authenticated [10]; authentication represents an additional protocol expense.

It is worthwhile to note the fraction of key sent by A which remains after error reconciliation and privacy amplification for each of the schemes discussed here. We label this fraction $R_{k}$ [12] and find

$$
R_{k}=\eta_{p}\left(\mu_{r}-\eta_{e} \frac{p_{o}}{p_{d}}\right)
$$

where $p_{o}$ is the measured error probability per bit in the key (before error reconciliation), and $\mu_{r}$ is the fraction of B's key material that remains after error reconciliation, and depends on $p_{o}$. The fraction of the distributed key that must be discarded through privacy maintenance is given by $\eta_{e} p_{o} / p_{d}$. Although an additional amount of key must be discarded if weak coherent states of light are used, this issue is disregarded in the discussion because $\bar{n}$ can be made arbitrarily small (in theory at least), and because other states of light can be employed that are not susceptible to this type of attack (e.g, entangled photon sources 13,14, or other single-photon states [4]).

Consider the (typical) fiber based BB84 implementation described above, and assume that the PM transmission is unity. This implementation has a receiver efficiency $\eta_{p(b b 84)}=1 / 4$ [1]]: $1 / 2$ from the 50:50 BS at the input of B's delay element and $1 / 2$ due to the random bases choice that is crucial to BB84 security. The $\eta_{e}$ and $p_{d}$ depend on the type of attack used by $\mathbf{E}$. The most powerful, and yet realistic, attack that has been devised for this scheme is for $\mathbf{E}$ to intercept and resend the photon pulses in the Breidbart Basis [7]. With this type of attack, $\eta_{e} \leq 0.585$ and $p_{d}=1 / 4$. If weak coherent pulses were used to transmit the key, another fraction $\bar{n} / 4$ of the key is also potentially compromised to $\mathbf{E}$, where $\bar{n} \ll 1$ is the average number of photons per superposition.

The IWY and BLT schemes accomplish key exchange in a similar manner. That is, superposed photon pulses (single-photon or weak-coherent states) are transmitted from A to B. B's delay element is adjusted so that a phase difference $\Delta \phi \in\{0, \pi\}$ between adjacent pulses in a superposition always cause a photodetection at a particular detector. After photodetections are observed, $\mathbf{B}$ tells $\mathbf{A}$ at what times they were observed relative to an accepted time standard. Because $\mathbf{A}$ knows what the phases of each superposition were, A knows which of B's detectors registered a photodetection.

As mentioned previously, $\eta_{p \|}=2 / 3$ while $\eta_{p+}=3 / 4$ for the implementations of BLT and IWY sketched in Figs. 1 and 2 respectively. The strongest attack that can be employed by $\mathbf{E}$ is likely an intercept/resend attack. However, unlike BB84, a Breidbart-like attack is not possible in IWY or BLT as the measurements are made in orthogonal bases. For these schemes, the strongest intercept/resend strategy is to build a receiver much like B, but to replace the first BS with a fast switch (we do not specify the switch technology, but merely state it is possible). In this manner, adjacent pulses can be made to overlap without loss of signal due to the first or last pulse in the superposition contributing less to the interference than the central pulses. If exactly one photon exists in the superposition $\mathbf{E}$ will measure one superposition phase difference $(\Delta \phi)$, but have no knowledge of the other remaining phase difference(s). Thus E's knowledge of the key for the superpositions that are intercepted and resent, and hence $\eta_{e}$ can be calculated as $\eta_{e \|}=1 / 2$ and $\eta_{e+}=1 / 3$ for the IWY and BLT respectively. The corresponding disturbances are $p_{d \|}=1 / 4$ and $p_{d+}=1 / 3$. If the effect of multiple photons in a superposition from the use of weak coherent states is included, $\mathbf{E}$ can know another fraction of the key equal to $\bar{n} / 4$ or $\bar{n} / 6$ for IWY or BLT respectively.

An important variation to either the IWY or BLT scheme is to add a BS and an extra delay element at the receiver as shown in (Fig. 14). For this system (denoted with $\oplus), \eta_{p \oplus}=5 / 8$ while $\eta_{e \oplus}=1 / 5$ is significantly 
reduced, and E's disturbance to the final key is increased to as much as $p_{d \oplus}=2 / 5$, if the additional BS is equally reflecting and transmitting.

With these considerations, $\eta_{p} \in\{1 / 4,2 / 3,3 / 4,5 / 8\}$ and $\eta_{e} / p_{d} \in\{2.34,2,1,1 / 2\}$ for $\{\mathrm{BB} 84$, IWY, BLT and BLT $\oplus\}$ respectively. The rate at which key is generated is proportional to $R_{k}$ and can be approximated from Fig. 5 where $R_{k}$ is plotted for the case of $\mu_{r}=$ $1+\left(1-p_{o}\right) \log _{2}\left(1-p_{o}\right)+p_{o} \log _{2}\left(p_{o}\right): 1$ minus the Shannon entropy.

In any case, in a practical QKD system, $R_{k}$ will be bounded by the values plotted in Fig. 5 for the four schemes at the same $p_{o}$. Figure 5 highlights the value of the relative schemes presented here. For $p_{o} \lesssim 0.13$, it is clear that BLT has a great advantage over the other three schemes: qubits are collected at a higher rate than for the other 3 schemes. For $p_{o} \gtrsim 0.13$, BLT $\oplus$ retains a significant advantage due to its reduced leakage of $\eta_{e}=1 / 5$, and disturbance $p_{d}=2 / 5$. These facts do not consider the added complexity of BLT $\oplus$ at $\mathbf{B}$ which includes the second delay element and additional detectors. For simplicity, efficiency, and protection against an intercept/resend attack by $\mathbf{E}$, BLT offers great advantages over BB84, IWY and BLT $\oplus$.

In conclusion, we have introduced two new quantum key distribution schemes for optical fiber systems based on the recently presented idea of Inoue, Waks and Yamamoto [i]. Our new schemes are more efficient and robust against eavesdropping than other schemes proposed to date (BLT $\oplus$ is more efficient than IWY for error rates $\gtrsim 2-3 \%$ ). In particular, BLT also has the advantage of being relatively easy and inexpensive to implement.

[1] K. Inoue, E. Waks and Y. Yamamoto (personal communication).

[2] C. H. Bennett and G. Brassard, in Proceedings of the IEEE International Conference on Computers, Signals, and Signal Processing, Bangalore, India, 1984 (IEEE, New York, 1984), pp. 175-179.

[3] J. D. Franson, Phys. Rev. A 44, 4552-4555 (1991).

[4] J. Kim, O. Benson, H. Kan and Y. Yamamoto, Nature 397, 500-503 (1999); P. Michler et al., Science 290, 2282 (2000); B. Lounis and W. E. Moerner, Nature 407, 491 (2000); Note: of the several single photon sources that have been proposed and demonstrated none of them are at a useful stage of development for QKD purposes at this time.

[5] R. J Hughes, G. L. Morgan and C. G. Peterson, J. Mod. Opt. 47, 533-547 (2000).

[6] A. Muller et al., Appl. Phys. Lett 70 793-795 (1997); P. D. Townsend, Nature 385, 47-49 (1997).

[7] C. H. Bennett et al., J. Cryptology 5, 3-25 (1992).

[8] G. Brassard and L. Salvail, Lect. Notes Comput. Sci. 765, 410 (1994).

[9] C. H. Bennett et al., IEEE Trans. on Inform. Th., 41 1915-1923 (1995).

[10] W. Diffie and M. E. Hellman, "Multi-user cryptographic techniques," Proceedings of AFIPS National Computer Conference, 109-112 (1976); R. L. Rivest, A. Shamir and L. M. Adleman, "A method for obtaining digitalsignatures and public-key cryptosystems," Communications of the ACM, 21, 120-126 (1978); C. Mitchell, F. Piper and P. Wild, "Digital Signatures," G. J. Simmons (Ed.), Contemporary Cryptography: The Science of Information Integrity, 325-378, IEEE Press, 1992.

[11] The BB84 protocol efficiency can be idealized to $\eta_{p(b b 84)}=1 / 2$ if $\mathbf{B}$ utilizes a switched receiver. We do not specify the technology, but note that it is possible.

[12] The privacy amplification key-rate specified here relates how much key must be removed irrespective of the additonal amount of key lost during error reconciliation.

[13] A. Ekert, Phys. Rev. Lett. 67, 661-663 (1991).

[14] D. S. Naik et al., Phys. Rev. Lett. 84, 4733-4736 (2000).

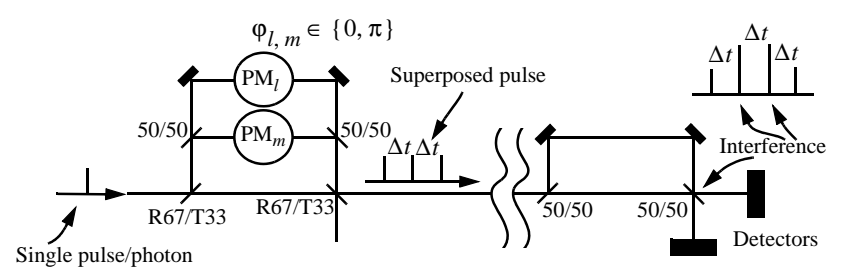

FIG. 1. The IWY parallel-delay element interferometer. Alice (A) is on left, Bob (B) on right. A controls all phase information $\left(\mathrm{PM}_{l}\right.$, and $\left.\mathrm{PM}_{m}\right)$, where the $l$ represents the "longer" path and $m$ represents the "medium" path (relative to the "short," or direct path). A randomly toggles $\phi_{m}$ and $\phi_{l}$ between values of 0 and $\pi$.

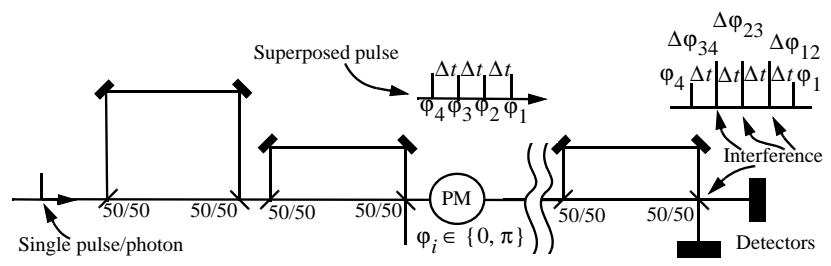

FIG. 2. The BLT series-delay element interferometer. A is on left, $\mathbf{B}$ on right. $\mathbf{A}$ controls all phase information by randomly adding a phase value of 0 or $\pi$ to each of the four temporal superposition locations.

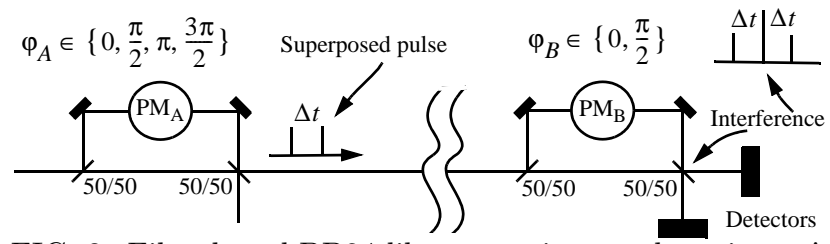

FIG. 3. Fiber based BB84-like transmitter and receiver. A is on left, $\mathbf{B}$ on right. The phase modulator at $\mathbf{A}$ is randomly toggled between one of four phases: $\phi_{A} \in\{0, \pi / 2, \pi, 3 \pi / 2\}$, and at $\mathbf{B}$ between one of two phases: $\phi_{B} \in\{0, \pi / 2\}$. 


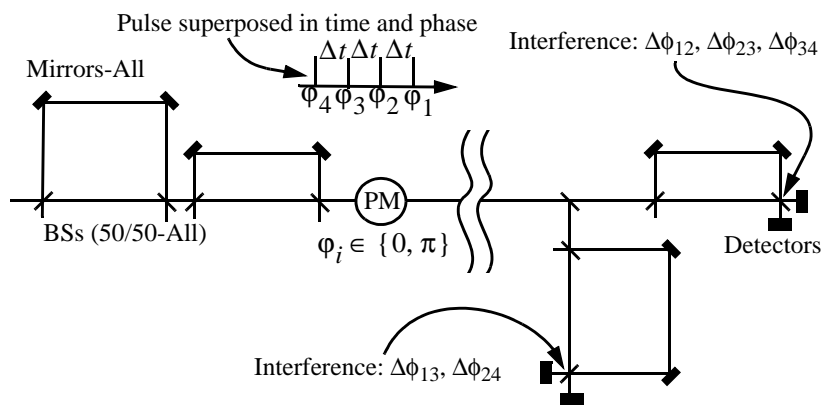

FIG. 4. A BLT series-delay interferometer variation with increased disturbance and reduced potential eavedropper information. A is on left, $\mathbf{B}$ on right. This system increases the eavesdropping disturbance to as much as $2 / 5$ and reduces E's information on the quantum key to a maximum of $1 / 5$.

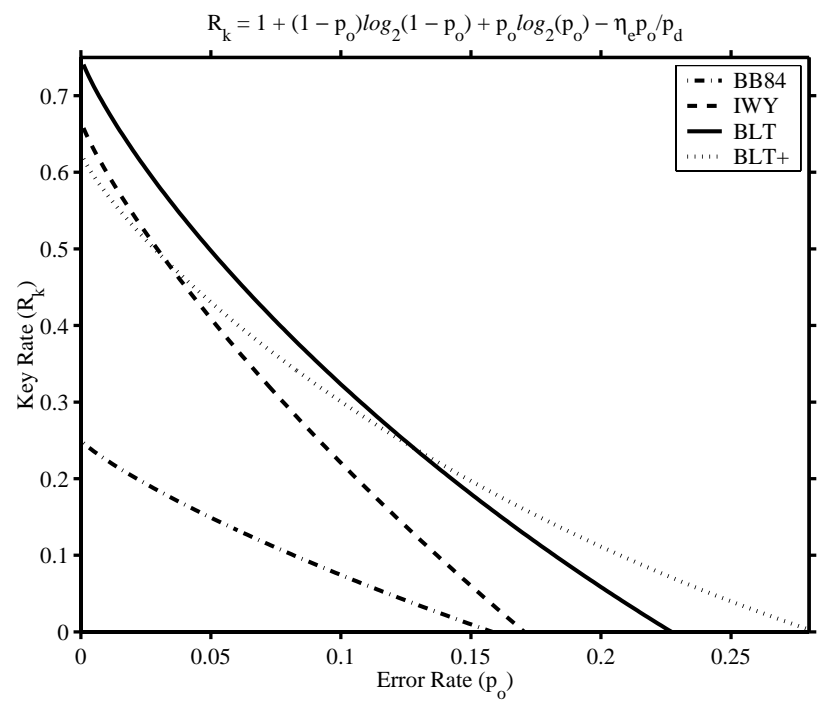

FIG. 5. Fraction of transmitted qubits which contribute to private key when privacy amplification is considered. This plot clearly shows that the series element idea has a great advantage over BB84, IWY, or BLT $\oplus$ for error rates les than about 0.13 . (The values presented here assume true single-photon QKD and do not consider weak coherent attack strategies, or the amount of key lost through error reconciliation.) 